\title{
SÍNTESE DE 5-NITRO-ISATINA E 5-CLORO-ISATINA A PARTIR DA ISONITROSOACETANILIDA ${ }^{\sharp}$
}

Bianca N. M. da Silva*, Renato S. Bastos, Bárbara V. Silva e Angelo C. Pinto

Instituto de Química, Universidade Federal do Rio de Janeiro, Cidade Universitária, CT, Bl. A, 21941-909 Rio de Janeiro - RJ, Brasil

Recebido em 11/6/10; aceito em 10/10/10; publicado na web em 8/11/10

PREPARATION OF 5-NITROISATIN AND 5-CHLOROISATIN FROM ISONITROSOACETANILIDE. This article describes the preparation of 5-nitroisatin and of 5-chloroisatin from isonitrosoacetanilide in a single step, using readily available and inexpensive reagents. These reactions require around 90 minutes and may be carried out as an undergraduate experiment, providing an opportunity to discuss the electrophilic aromatic substitution mechanism, as well as spectroscopic techniques for product identification.

Keywords: 5-nitroisatin; 5-chloroisatin; isonitrosoacetanilide.

\section{INTRODUÇÃO}

A isatina (1, Esquema 1) (1- $H$-indol-2,3-diona) é um heterociclo muito utilizado em síntese orgânica, devido às possibilidades de modificação na sua estrutura. Possui duas carbonilas com reatividades distintas, uma cetônica [C-3] e outra amídica [C-2], um grupo N-H suscetível a reações de alquilação e acilação, e um anel aromático que pode sofrer reações de substituição eletrofílica nas posições C-5 e C-7. A isatina é encontrada em plantas do gênero Isatis, por exemplo, nas espécies Calanthe discolor Lindl. e Coroupita guianenses Aubl., na secreção da glândula parótida de sapos do gênero $B u f o^{2}$ e, ainda, distribuída em diferentes regiões do cérebro e em fluídos corporais de seres humanos. ${ }^{3}$

O método de Sandmeyer é o mais utilizado para a obtenção da isatina e de seus derivados. Nesta metodologia, a anilina (2) ou as anilinas substituídas (Esquema 1, etapa I) reage (m) com hidrato de cloral e sulfato de hidroxilamina (ou outro sal de hidroxilamina), na presença de uma solução saturada de sulfato de sódio, para formar isonitrosoacetanilida(s) (3). Em seguida, a(s) isonitrosoacetanilida(s) 3 sofre $(\mathrm{m})$ ciclização em ácido sulfúrico concentrado, para formar a(s) respectiva(s) isatina(s) (Esquema I, etapa II). ${ }^{4,5}$ Outras metodologias para a síntese de isatinas, como as de Stolle, ${ }^{6}$ Martinet, ${ }^{7}$ Gassman $^{8}$ e Pinto, ${ }^{9}$ são descritas na literatura.

A isatina e seus derivados são conhecidos na literatura pela variedade de suas propriedades biológicas, sendo, também, frequentemente empregados como matéria-prima para a obtenção de compostos bioativos como, por exemplo, fármacos. ${ }^{1}$ Este é o caso do sunitinibe (4, Figura 1), aprovado, em 2006, pelo FDA (Food and Drugs Administration) para o tratamento de carcinoma de células renais (RCC) e de tumores do estroma gastrointestinal (GIST). ${ }^{10}$

Vine e colaboradores ${ }^{11}$ mostraram que algumas isatinas substituídas apresentam ação anticancerígena sobre células humanas de linfoma histiocítica (U937), principalmente as halogenadas e nitradas na posição 5. Na Figura 1 estão ilustrados exemplos de derivados contendo, ao mesmo tempo, os grupos nitro e bromo (5), apenas bromo (6) e flúor (7). Os valores de $\mathrm{CI}_{50}$ (concentração que causa $50 \%$ de inibição do crescimento das células) destas isatinas são bem menores do que os observados para a isatina (1).

A escolha de reagentes de preço baixo e menos agressivos ao meio ambiente são dois dos requisitos mais procurados pelos químicos orgânicos de síntese. Estes requisitos são quase que obrigatórios quando se trata do emprego de reagentes em disciplinas experimentais

*e-mail: bianca_qnascimento@yahoo.com.br

\# Artigo em homenagem ao Prof. Hans Viertler

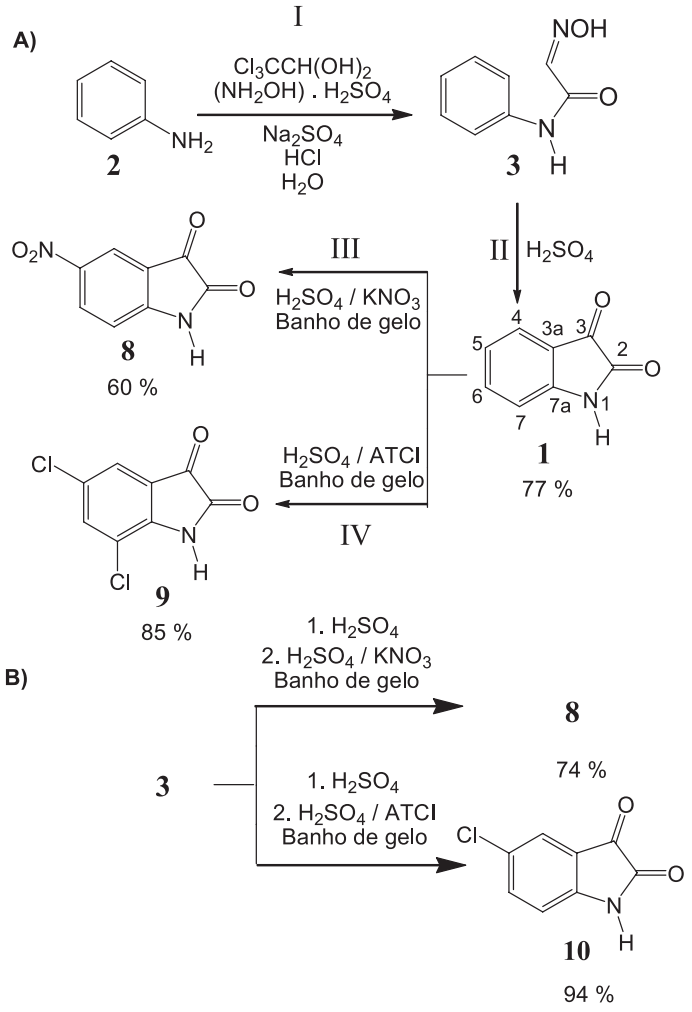

Esquema 1. (A) Obtenção da isonitrosoacetanilida a partir da anilina (etapa I), ciclização da isonitrosoacetanilida levando à formação da isatina (etapa II), nitração da isatina (etapa III), cloração da isatina (etapa IV). (B) Método de obtenção da 5-nitro-isatina (8) e 5-cloro-isatina (10) por única etapa através da isonitrosoacetanilida (3)

de Cursos de Graduação em Química e de áreas afins.

Neste artigo, descreve-se a preparação de 5-cloro-isatina e 5-nitroisatina obtidas diretamente de isonitrosoacetanilida, através de uma metodologia simples e eficiente, partindo-se de reagentes baratos e de baixa toxicidade.

\section{PARTE EXPERIMENTAL}

\section{Materiais e métodos}

Os produtos obtidos em cada etapa de reação foram caracteriza- 
<smiles>CCN(CC)CCNC(=O)c1c(C)[nH]c(C=C2C(=O)Nc3ccc(F)cc32)c1C</smiles><smiles>O=C1Nc2cc(Br)c([N+](=O)[O-])cc2C1=O</smiles>

$\mathrm{CI}_{50} 17,1 \mu \mathrm{M}$

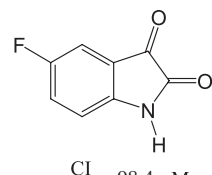

7

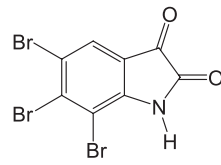

$\mathrm{CI}_{50} 6,76 \mu \mathrm{M}$

6

$\mathrm{CI}_{50} 565 \mu \mathrm{M}$

Figura 1. Estrutura do sunitinibe (4) e dos derivados da isatina nitrados e halogenados (5-7) acompanhados dos seus respectivos valores de $\mathrm{CI}_{50}$

dos por ponto de fusão, espectroscopia na região do infravermelho (IV) e ressonância magnética nuclear $(\mathrm{RMN})$ de hidrogênio $\left({ }^{1} \mathrm{H}\right)$ e de carbono $\left({ }^{13} \mathrm{C}\right)$.

Os espectros na região do infravermelho (IV) foram obtidos em aparelho Nicolet Magna-IR 760, em pastilhas de brometo de potássio anidro $(\mathrm{KBr})$. Os valores das absorções são representados em número de onda, e a unidade utilizada é o centímetro recíproco $\left(\mathrm{cm}^{-1}\right)$.

Os espectros de RMN de ${ }^{1} \mathrm{H}$ e ${ }^{13} \mathrm{C}$ foram obtidos em aparelho Bruker DRX em frequências de 200 e $50 \mathrm{MHz}$, respectivamente. Foram utilizados como solventes, uma mistura de clorofórmio $\left(\mathrm{CDCl}_{3}\right)$ e dimetilsulfóxido (DMSO-d $\mathrm{d}_{6}$ ).

As medidas de absorbância foram obtidas empregando-se espectrofotômetro UV-visível Varian modelo Cary 1E, utilizando cela de $1 \mathrm{~cm}$ de caminho óptico. As concentrações das soluções, preparadas em metanol, foram de $10^{-4} \mathrm{M}$.

Os pontos de fusão dos produtos foram determinados em aparelho Mel-Temp, utilizando capilares de vidro, e os seus valores não foram corrigidos.

\section{Preparo da isonitrosoacetanilida (3)}

A um balão de $500 \mathrm{~mL}$ foram adicionados $130 \mathrm{~g}$ de sulfato de sódio $(0,915 \mathrm{~mol}), 120 \mathrm{~mL}$ de água destilada, $18 \mathrm{~g}$ de hidrato de cloral $(0,109 \mathrm{~mol}), 13 \mathrm{~g}$ de sulfato de hidroxilamina $(0,079 \mathrm{~mol})$ dissolvidos em $50 \mathrm{~mL}$ de água destilada, $0,1 \mathrm{~mol}$ de anilina em 60 $\mathrm{mL}$ de água destilada e $8,6 \mathrm{~mL}$ de ácido clorídrico concentrado. $\mathrm{O}$ banho de óleo foi aquecido gradualmente até $70^{\circ} \mathrm{C}$, com posterior adição de $100 \mathrm{~mL}$ de álcool etílico. O meio de reação foi mantido sob agitação magnética e refluxo durante $4 \mathrm{~h}$. Após este período, a mistura foi vertida em banho de gelo, filtrada em funil de Büchner, à pressão reduzida, e lavada com água gelada. ${ }^{5} \mathrm{~A}$ isonitrosoacetanilida foi obtida em $87 \%$ de rendimento.

\section{Preparo da isatina (1)}

Em um erlenmeyer, a isonitrosoacetanilida (3) (3,00 g, $18 \mathrm{mmol})$ foi adicionada lentamente ao ácido sulfúrico concentrado $(9 \mathrm{~mL})$ sob agitação magnética, à temperatura ambiente. $\mathrm{O}$ final da reação foi identificado pela elevação da temperatura e pela gradual mudança de coloração de vermelho à púrpura. A solução foi, então, vertida sobre gelo picado e o precipitado formado foi filtrado em funil de Büchner, à pressão reduzida, e lavado com água gelada. ${ }^{4,5} \mathrm{~A}$ isatina foi obtida em $77 \%$ de rendimento.

Preparo da 5-nitro-isatina (8) e da 5,7-dicloro-isatina (9)

Preparo da 5-nitro-isatina (8)

A um erlenmeyer foi adicionada uma solução contendo $20 \mathrm{mmol}$ $(3,00 \mathrm{~g})$ da isatina (1), previamente preparada pelo método de Sandmeyer, modificado por Marvel e Hiers, completamente solubilizada em $40 \mathrm{~mL}$ de ácido sulfúrico concentrado $\left(\mathrm{H}_{2} \mathrm{SO}_{4}\right)$. Em seguida, o erlenmeyer com esta solução foi resfriada a $0{ }^{\circ} \mathrm{C}$, em banho de gelo, adicionando-se uma solução de $19 \mathrm{mmol}(1,92 \mathrm{~g})$ de nitrato de potássio $\left(\mathrm{KNO}_{3}\right)$ em $4 \mathrm{~mL}$ de ácido sulfúrico concentrado $\left(\mathrm{H}_{2} \mathrm{SO}_{4}\right)$. A reação foi mantida sob agitação magnética, a $0{ }^{\circ} \mathrm{C}$, durante 30 min. Decorrido este tempo, a mistura foi vertida sobre gelo picado e o precipitado amarelo formado foi filtrado, à pressão reduzida, em funil de Büchner. ${ }^{12}$ A 5-nitro-isatina foi obtida em $60 \%$ de rendimento.

\section{Preparo da 5,7-dicloro-isatina (9)}

Em um erlenmeyer colocou-se 4,64 g (20 mmol) de ácido tricloroisocianúrico (ATCI) e $12 \mathrm{~mL}$ de ácido sulfúrico. O erlenmeyer foi mantido a $0{ }^{\circ} \mathrm{C}$ em banho de gelo, e à solução de $\mathrm{ATCI}$ em $\mathrm{H}_{2} \mathrm{SO}_{4}$ adicionou-se 2,94 g (20 mmol) de isatina. Posteriormente, manteve-se a suspensão sob agitação magnética por 30 min à temperatura ambiente. A mistura foi vertida sobre gelo picado. O precipitado obtido foi filtrado, à pressão reduzida, em funil de Büchner e lavado com água gelada. ${ }^{13}$ A 5,7-dicloro-isatina foi obtida em $85 \%$ de rendimento.

\section{Preparo da 5-nitro-isatina e da 5-cloro-isatina por reação direta da isonitrosoacetanilida}

Preparo da 5-nitro-isatina (8) diretamente da isonitrosoacetanilida

A um erlenmeyer com $10 \mathrm{~mL}$ de ácido sulfúrico concentrado, mantido sob agitação magnética, adicionou-se lentamente 3,28 g (20 mmol) da isonitrosoacetanilida (3). Como a reação de ciclização é exotérmica, introduz-se o termômetro na solução, como controle para não deixar a temperatura ultrapassar $60^{\circ} \mathrm{C}$. Após a solução adquirir coloração púrpura, o erlenmeyer foi imerso em banho de gelo (temperatura controlada entre 0 e $3{ }^{\circ} \mathrm{C}$ ), e a este se gotejou, durante $1 \mathrm{~h}$, uma solução de $1,92 \mathrm{~g}(19 \mathrm{mmol})$ de nitrato de potássio $\left(\mathrm{KNO}_{3}\right)$ em $19 \mathrm{~mL}$ de ácido sulfúrico. A mistura foi, então, vertida sobre gelo picado e o precipitado amarelo formado foi filtrado, à pressão reduzida, em funil de Büchner, e lavado exaustivamente com água gelada. A 5-nitro-isatina foi obtida em $74 \%$ de rendimento.

\section{Preparo da 5-cloro-isatina (10) diretamente da isonitrosoacetanilida}

Em erlenmeyer mantido em banho de gelo adicionou-se a isonitrosoacetanilida $(2,94 \mathrm{~g}, 17,9 \mathrm{mmol})$ e $12 \mathrm{~mL}$ de ácido sulfúrico concentrado $\left(\mathrm{H}_{2} \mathrm{SO}_{4}\right)$. Em seguida, adicionou-se 2,90 g (12,4 mmol) de ATCI. Quinze minutos após a adição do ATCI, o banho de gelo foi removido, e a mistura foi mantida à temperatura ambiente, sob agitação magnética, durante $30 \mathrm{~min}$. A mistura foi, então, vertida sobre gelo picado e o precipitado formado foi filtrado à pressão reduzida, em funil de Büchner, e lavado com água gelada. A 5-cloro-isatina foi obtida em $94 \%$ de rendimento.

\section{RESULTADOS E DISCUSSÃO}

A primeira parte do trabalho consistiu na preparação da isatina (1) através do método clássico de Sandmeyer, da 5-nitro-isatina (8) e da 5,7-dicloro-isatina (9) através de reações de substituição eletrofílica da isatina com nitrato de potássio em ácido sulfúrico e ácido 
tricloroisocianúrico (ATCI, 11 - Figura 2), respectivamente, seguindo procedimentos da literatura (Esquema 1A).<smiles>O=c1n(Cl)c(=O)n(Cl)c(=O)n1Cl</smiles><smiles>O=c1n(Cl)c(=O)n(Cl)c(=O)n1Cl</smiles>

11a<smiles>O=c1n(Cl)c(=O)n(Cl)c(=O)n1Cl</smiles>

11b<smiles>O=c1n(Cl)c(=O)n(Cl)c(=O)n1Cl</smiles>

$11 \mathrm{c}$
Figura 2. Espécies supereletrofílicas (11a, 11 b e 11c) formadas da reação entre $\mathrm{ATCI}$ e $\mathrm{H}_{2} \mathrm{SO}_{4}$

Geralmente as aulas experimentais são divididas em duas partes distintas. Na primeira o professor faz considerações sobre a toxidez e os cuidados que devem ser tomados com a manipulação dos reagentes e sobre o mecanismo de reação. Na segunda parte os estudantes fazem o experimento, e quanto mais rápido este for, mais o professor dispõe de tempo para explicações. Logo, para que isto aconteça é necessário que se procure eliminar certas etapas ou procedimentos de reação, quando isto for possível. Este foi o motivo que nos levou a propor a preparação direta da 5-cloro e da 5-nitro-isatina a partir da isonitrosoacetanilida (Esquema 1B), ao invés de fazer a cloração e a nitração da isatina.

A nitração e a cloração da isonitrosoacetanilida com $\mathrm{KNO}_{3} / \mathrm{H}_{2} \mathrm{SO}_{4}$ e ATCI/ $\mathrm{H}_{2} \mathrm{SO}_{4}$ forneceram os compostos $\mathbf{8}$ e 10, respectivamente, em bons rendimentos (Esquema 1B). A 5-nitro-isatina foi obtida em rendimento superior ao obtido através do método clássico de preparação (nitração da isatina). Para surpresa nossa, na reação de cloração da isonitrosoacetanilida obteve-se a 5-cloro-isatina (10) ao invés da 5,7-dicloro-isatina (9). A obtenção da isatina monoclorada, neste caso, apesar de não ser o produto que, em princípio, esperávamos, tem grandes vantagens porque não exige o controle de temperatura, que é necessário quando se parte da isatina. ${ }^{13}$

Uma possível explicação para a monocloração quando se parte da isonitrosoacetanilida pode ser devida à diferença de solubilidade do meio de reação, que talvez faça com que $\mathbf{1 0}$ fique em suspensão e, assim, não sofra a segunda reação de cloração.

As reações de cloração e de nitração de substratos aromáticos ocorrem, via de regra, através de reações de substituição eletrofílica aromática, um dos temas muito abordados em química orgânica, nos cursos de graduação. Em uma reação deste tipo, um eletrófilo sofre $\mathrm{o}$ ataque dos elétrons pi do anel aromático levando à formação do intermediário de Wheland que, em seguida, perde um próton para restaurar a aromaticidade do sistema. ${ }^{14}$

Se o anel aromático já tiver um substituinte, a adição do eletrófilo pode ocorrer nas posições ipso, orto, meta ou para em relação ao substituinte, dependendo da natureza deste último. No caso da isatina, as posições C-5 e C-7, orto e para em relação ao nitrogênio do heterociclo, são as mais favorecidas em reações de substituição eletrofílica.

A literatura descreve que o ATCI $\mathbf{1 1}^{15} \mathrm{em}$ ácido sulfúrico forma espécies supereletrofílicas, primordiais para a transferência de cloro eletrofílico ("Cl+") ao anel aromático da isatina (Figura 2). ${ }^{16}$ Vasconcellos e Lima Junior ${ }^{17}$ mostraram que a protonação diminui a energia do LUMO do ATCI mono, di e tri-protonados (11a, 11b e 11c), aproximando-os da energia do HOMO do nucleófilo.
Na reação de nitração do anel aromático, é normalmente aceito que a espécie " $\mathrm{NO}_{2}{ }^{+}$" atue como eletrófilo. Entretanto, este mecanismo vem sendo questionado por alguns autores que postulam que a reação ocorra via transferência de elétrons. ${ }^{18}$

\section{Caracterização dos produtos}

Como a isonitrosoacetanilida apresenta um plano de simetria, no espectro de RMN ${ }^{1} \mathrm{H}$ (Figura 3) os sinais dos hidrogênios aromáticos, H-1 e H-5, H-2 e H-4, apresentam os mesmos deslocamentos químicos. ${ }^{19}$ Estes hidrogênios acoplam entre si, e aparecem como um dubleto em 7,05 ppm (H-1 e H-5), tripleto em 6,67 ppm (H-2 e H-4) e tripleto em 6,45 ppm (H-3). A constante de acoplamento $(J)$ para os três sinais é de $8 \mathrm{~Hz}$. O sinal do hidrogênio de amida é largo e aparece em 9,09 ppm, enquanto o hidrogênio ligado ao oxigênio (grupamento oxima) é bem mais desprotegido, aparecendo em 11,39 ppm.<smiles>NC(=O)C(=O)Nc1ccccc1</smiles>

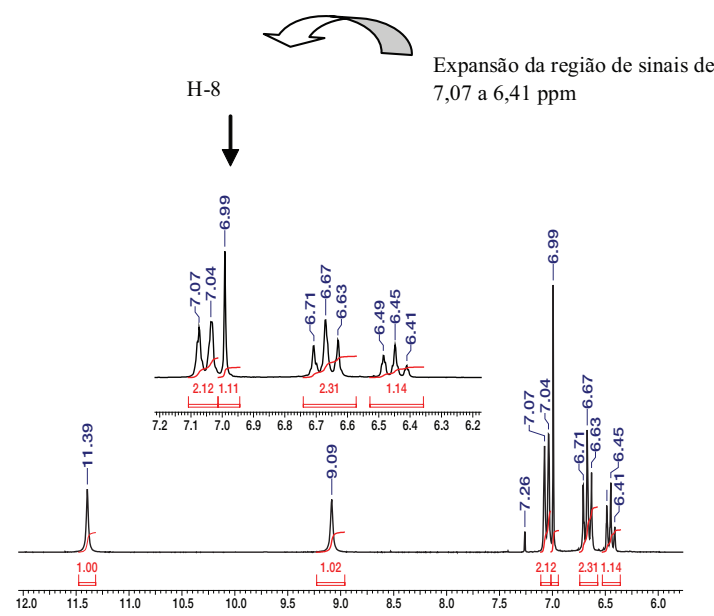

Figura 3. Espectro de $R M N^{l} H$ da isonitrosoacetanilida

No RMN de ${ }^{13} \mathrm{C}$ (Figura 1S, material suplementar) foram observados seis sinais, embora a isonitrosoacetanilida possua oito átomos de carbonos, devido à simetria da molécula. ${ }^{19}$

O padrão de substituição do anel aromático da isatina no espectro de $\mathrm{RMN}{ }^{1} \mathrm{H}$ é bem distinto do da isonitrosoacetanilida. Para a isatina são observados os quatro sinais de hidrogênio ligados aos carbonos do anel aromático. Dois dubletos largos com $J=8 \mathrm{~Hz}$ em 7,48 e $6,89 \mathrm{ppm}$ referentes a $\mathrm{H}-4$ e H-7, respectivamente. Os sinais de $\mathrm{H}-5$ (7,04 ppm) e H-6 (7,57 ppm) desdobram-se em um triplo dubleto com valores de $J=8$ (orto) e 2 (meta) $\mathrm{Hz}$ (Figura 4).

$\mathrm{O}$ espectro de $\mathrm{RMN}$ de ${ }^{13} \mathrm{C}$ da isatina (Figura $2 \mathrm{~S}$, material suplementar) mostra oito sinais, seis correspondentes ao anel aromático (112151 ppm) e dois às carbonilas de cetona (184 ppm) e amida (159 ppm).

Na Figura 3S, material suplementar, são apresentados os espectros de $\mathrm{RMN}{ }^{1} \mathrm{H}$, mostrando apenas a região expandida dos sinais correspondentes aos hidrogênios do anel aromático, da 5-nitro (A), 5-cloro (B) e 5,7-dicloro (C) isatina. A análise desta região do espectro já é suficiente para diferenciá-las entre si e também do material de partida.

Todos apresentam um dubleto mais protegido em 7,06 (A); 6,83 (B) e 6,87 (C) ppm, correspondentes ao H-7 da 5-nitro-isatina e 5-cloro-isatina, e H-6 da 5,7-dicloro-isatina, respectivamente. Estes dubletos apresentam $J$ de $8 \mathrm{~Hz}$, indicando um acoplamento orto entre H-7 e H-6 nos espectros A e B, enquanto que na 5,7-dicloro-isatina 


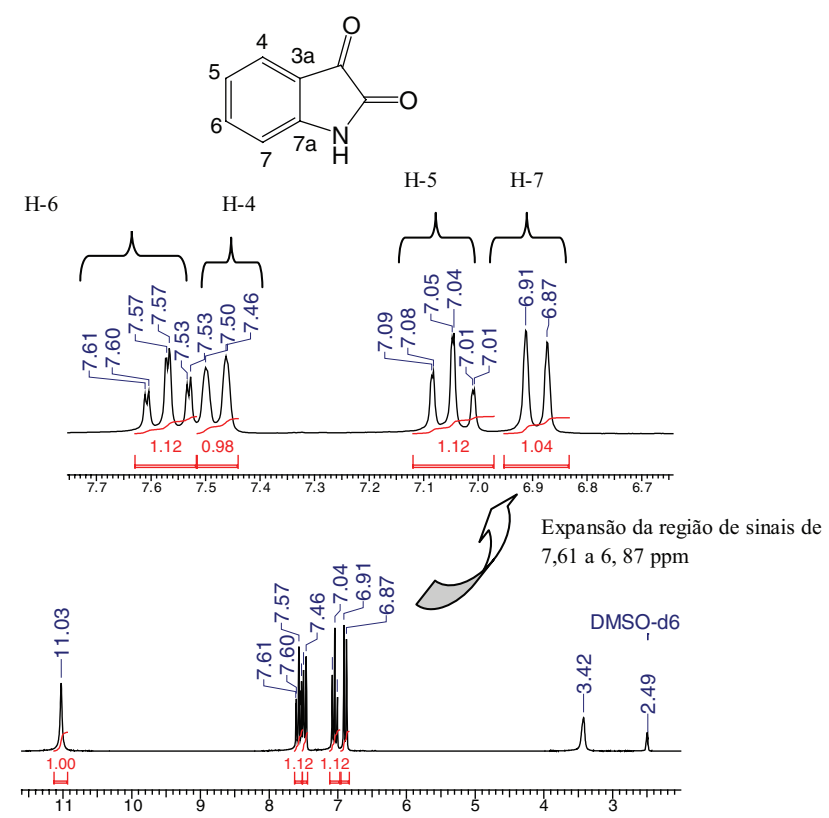

Figura 4. Espectro de $R M N^{1} H$ da isatina

é observado um dubleto em 6,87 ppm com $J$ de $2 \mathrm{~Hz}$, indicando acoplamento meta entre H-6 e H-4.

O H-6 da 5-nitro-isatina aparece como um dubleto em 8,37 ppm com $J=8 \mathrm{~Hz}$, e H-4 como um singleto em 8,20 ppm, não se observando o acoplamento meta.

A espectroscopia na região do infravermelho ${ }^{19,20}$ também foi utilizada para a caracterização dos produtos (Figuras $6 \mathrm{~S}, 8 \mathrm{~S}, 12 \mathrm{~S}$, $15 \mathrm{~S}$ e $18 \mathrm{~S}$, material suplementar). As absorções dos grupos hidroxila e $\mathrm{NH}$ da isonitrosoacetanilida, substância $\mathbf{3}$, foram observadas por volta de $3300 \mathrm{~cm}^{-1}$. Para as isatinas $(\mathbf{8 , 9} \mathrm{e} \mathrm{10})$, as bandas referentes à carbonila de cetona e amida estão por volta de $1700 \mathrm{~cm}^{-1}$. A absorção do estiramento da ligação $\mathrm{Ar}-\mathrm{Cl}$ nos compostos $\mathbf{9}$ e $\mathbf{1 0}$ aparece em torno de $1100 \mathrm{~cm}^{-1}$. O composto 8 apresentou duas absorções características da ligação Ar-NO ${ }_{2}$, em torno de 1565 e $1336 \mathrm{~cm}^{-1} \cdot{ }^{19,21}$

Como pode ser observado na Figura $4 \mathrm{~S}$ (material suplementar), o espectro de UV de cada substância, em metanol, comprova a presença de sistemas conjugados, sendo observadas bandas com comprimentos de onda máximo em nm $\left(\lambda_{\text {máx }}\right)$ e absortividade $\mathrm{em} \mathrm{L} \mathrm{cm}^{-1} \mathrm{~mol}^{-1}(\varepsilon) 297$ (ع 2370), 279 (ع 17920), 318 (ع 9340), 225 (ع 4860) e 225 (ع 3370) para $\mathbf{1}, \mathbf{3}, \mathbf{8}, \mathbf{9}$ e $\mathbf{1 0}$, respectivamente. ${ }^{19,21}$

Devido à semelhança estrutural, os espectros de UV dos compostos 9 e 10 são quase sobrepostos.

\section{CONCLUSÃO}

Os métodos de preparação da 5-nitro-isatina (11) e da 5-cloroisatina (10) a partir da isonitrosoacetanilida, além do seu caráter didático, são adequados para aulas experimentais de graduação de cursos de Química e áreas afins, pois são reações relativamente rápidas e que utilizam materiais comercialmente disponíveis, de baixo custo, baixa toxicidade e fácil manipulação. As estruturas químicas destes compostos são facilmente caracterizadas por métodos espectroscópicos.

A preparação da 5-nitro-isatina (11) e da 5-cloro-isatina (10), a partir da isonitrosoacetanilida, resultou em economia de tempo e de reagentes.

A obtenção da 5-cloro-isatina (10) pode ser utilizada como material de partida tanto para a obtenção da 5,7-dicloro-isatina como para outras substâncias de interesse farmacológico. A principal vantagem para a preparação de $\mathbf{1 0}$ quando se parte da isonitrosoacetanilida é que se obtém o produto de monocloração.
O ácido tricloroisocianúrico (ATCI) é um excelente agente de cloração de anéis aromáticos. Além de ser um produto muito barato e de baixa toxicidade quando comparado a alguns reagentes usados para cloração como, por exemplo, a $N$-clorosuccinamida (NCS), pode ser encontrado em lojas que comercializam produtos para o tratamento de água de piscina. ${ }^{15}$

\section{MATERIAL SUPLEMENTAR}

Está disponível em http://quimicanova.sbq.org.br, na forma de arquivo PDF, com acesso livre.

\section{REFERÊNCIAS}

1. Silva, J. F. M.; Garden, S. J.; Pinto, A. C.; J. Braz. Chem. Soc. 2001, 12, 273.

2. Wei, L.; Wang, Q.; Liu, X.; Yaowu Fenxi Zazhi 1982, 2, 288. (CA 98:95726b).

3. Watkins, P.; Clow, A.; Glover, V.; Halket, J.; Przyborowska, A.; Sandler, M.; Neurochem. Inter. 1990, 17, 321.

4. Sandmeyer, T.; Helv. Chim. Acta 1919, 2, 234.

5. Marvel, C. S.; Hiers, G. S.; Org. Synth. Coll. 1941, 1, 327; Garden, S. J.; Torres, J. C.; Ferreira, A. A.; Silva, R. B.; Pinto, A. C.; Tetrahedron Lett. 1997, 38, 1501.

6. Fukuda, Y.; Itoh, Y.; Nakatani, K.; Terashima, S.; Tetrahedron 1994, 50, 2793; Loloiu, G.; Maior, O.; Rev. Roum. Chim. 1997, 42, 67.

7. Taylor, A. J.; J. Chem. Res. (S) 1980, 347.

8. Gassman, P. G.; Cue Jr, B. W.; Luh, T. Y.; J. Org. Chem. 1977, 42, 1344.

9. Pinto, A. C.; Lapis, A. A. M.; Silva, B. V.; Bastos, R. S.; Dupont, J.; Neto, B. A. D.; Tetrahedron Lett. 2008, 49, 5639.

10. Sun, S.; Schiller, J. H.; Crit. Rev. Oncol. Hematol. 2007, 62, 93; Faivre, S.; Demetri, G.; Sargente, W.; Raymond, E.; Nature 2007, 6, 734; http:// www.portaldosfarmacos.ccs.ufrj.br/resenhas_sunitinib.html, acessada em Abril 2010.

11. Vine, K. L.; Locke, J. M.; Ranson, M.; Pyne, S. G.; Bremner, J. B.; Bioorg. Med. Chem. 2007, 15, 931; Vine, K. L.; Matesic, L.; Locke, J. M.; Ranson, M.; Skropeta, D.; Anti-Cancer Agents Med. Chem. 2009, 9, 397.

12. Calvery, H. O.; Noller, C. R.; Adams, C.; J. Am. Chem. Soc. 1925, 47, 3058.

13. Ribeiro, N. M.; Silva, B. V.; Violante, F. A.; Rezende, C. M.; Pinto, A. C.; Org. Prep. Proc. Int. 2005, 37, 265.

14. Clayden, J.; Greeves, N.; Warren, S.; Wothers, P.; Organic Chemistry, $5^{\text {th }}$ ed., Oxford University Press, 2007.

15. Tilstam, U.; Weinmann, H.; Org. Process. Res. Dev. 2002, 6, 384; Cunha, A. C.; Paixão, F. M.; Souza, M. C. B. V.; Ferreira, V. F.; Quim. Nova 2006, 29, 520; Bastos, R. S.; Cunha, A. S.; Silva, L. C.; Oliveira, C. C. P.; Rezende, C. M.; Pinto, A. C.; Quim. Nova 2008, 31, 172; Mendonça, G. F.; Mattos, M. C. S.; Quim. Nova 2008, 31, 798.

16. Mendonça, G. F.; Magalhães, R. R.; Esteves, P. M.; de Mattos, M. C. S.; J. Braz. Chem. Soc. 2005, 16, 695.

17. Vasconcellos, M. L. A. A.; Lima Jr., C. G.; Quim. Nova 2009, 32, 244.

18. Cardoso, S. P.; Carneiro, J. W. M.; Quim. Nova 2001, 24, 381.

19. Silverstein, R. M.; Bassler, G. C.; Morrill, T. C.; Identificação Espectrométrica de Compostos Orgânicos, LTC - Livros Técnicos e Científicos Ed. S.A.: Rio de Janeiro, 2007, tradução de R. B. de Alencastro; Pretsch, E.; Clerc, T.; Seibl, J.; Simon, W.; Tables of Spectral Data for Structure Determination of Organic Compounds, Springer-Verlag: New York, $2^{\text {nd }}$ ed., 1989.

20. Barbosa, L. C. A.; Espectroscopia no infravermelho na caracterização de compostos orgânicos, Ed. da UFV: Viçosa, 2007.

21. Scott, A. I.; Interpretation of the Ultraviolet Spectra of Natural Products, Pergamon Press: New York, 1964. 